\title{
JORDAN LEFT DERIVATIONS IN FULL AND UPPER TRIANGULAR MATRIX RINGS*
}

\author{
XIAO WEI XU ${ }^{\dagger}$ AND HONG YING ZHANG ${ }^{\dagger}$
}

\begin{abstract}
In this paper, left derivations and Jordan left derivations in full and upper triangular matrix rings over unital associative rings are characterized.
\end{abstract}

Key words. Left derivations, Jordan left derivations, Full matrix rings, Triangular matrix rings.

AMS subject classifications. 16S50, 16W25.

1. Introduction. Let $R$ be an associative ring. An additive mapping $\delta: R \rightarrow M$ from $R$ into a bimodule ${ }_{R} M_{R}$ is called a module derivation if $\delta(x y)=\delta(x) y+x \delta(y)$ holds for all $x, y \in R$. Particularly, the module derivation from $R$ into its regular bimodule ${ }_{R} R_{R}$ is well known as the ring derivation (usually called derivation). Obviously, the concept of module derivations depends heavily on the bimodule structure of $M$, i.e., if $M$ is a left $R$-module but not a right $R$-module, this concept will not happen. However, a small modification can lead a new concept, that is, the concept of module left derivations. Exactly, an additive mapping $\delta$ from a ring $R$ into its left module $R_{R} M$ is called a module left derivation if $\delta(x y)=x \delta(y)+y \delta(x)$ holds for all $x, y \in R$. Particularly, a module left derivation from $R$ into its left regular module ${ }_{R} R$ is called a ring left derivation (usually called a left derivation).

The concept of (module) left derivations appeared in Brešar and Vukman [8] at first. They obtained that a left derivation in a prime ring must be zero, that a left derivation in a semiprime ring must be a derivation such that its range is contained in the center, and that a continuous linear left derivation in a Banach algebra $A$ must map $A$ into its Jacobson radical $\operatorname{Rad}(A)$. Since left derivations act in accord with derivations in a commutative ring, the result on Banach algebra by Brešar and Vukman can be seen as a generalization of the one by Singer and Wermer [22] which states that a continuous linear derivation in a commutative Banach algebra $A$ must map $A$ into its Jacobson radical $\operatorname{Rad}(A)$.

Since Brešar and Vukman initiated the study of left derivations in noncom-

${ }^{*}$ Received by the editors on April 20, 2010. Accepted for publication on October 22, 2010. Handling Editor: Robert Guralnick.

${ }^{\dagger}$ College of Mathematics, Jilin University, Changchun 130012, PR China (xuxw@jlu.edu.cn, zlzxzhy@163.com). Supported by the NNSF of China (No. 10871023 and No. 11071097), 211 Project, 985 Project and the Basic Foundation for Science Research from Jilin University. 
mutative rings, many related results have appeared for both Banach algebras (for example, see $[12,14,15,16,20,21,23,24]$ ) and prime rings (for example, see $[1,3,4,5,10,13,24,25,26])$. However, in this paper, we will concerned ourselves with (Jordan) left derivations in full and upper triangular matrix rings over unital associative rings.

Recall that an additive mapping $\delta: R \rightarrow M$ from a ring $R$ into its bimodule ${ }_{R} M_{R}$ is called a module Jordan derivation if $\delta\left(x^{2}\right)=\delta(x) x+x \delta(x)$ holds for all $x \in R$. Particularly, a module Jordan derivation from $R$ into its regular bimodule ${ }_{R} R_{R}$ is called a ring Jordan derivation (usually called a Jordan derivation). Similarly, an additive mapping $\delta$ from a ring $R$ into its left module ${ }_{R} M$ is called a module Jordan left derivation if $\delta\left(x^{2}\right)=2 x \delta(x)$ holds for all $x \in R$. Particularly, a module Jordan left derivation from $R$ into its left regular module ${ }_{R} R$ is called a ring Jordan left derivation (usually called a Jordan left derivation). For both Banach algebras and prime rings, Jordan left derivations have been studied broadly.

On the other hand, (Jordan) derivations in full and upper triangular matrix rings over unital rings have been characterized (see $[2,6,7,9,17,18,19]$ ). This short note will characterize (Jordan) left derivations in full and upper triangular matrix rings over unital rings.

Unless stated otherwise, $R$ always denotes a unital associative ring with left $R$ module ${ }_{R} V$. Let $\mathbb{M}_{n}(R)$ and $\mathbb{T}_{n}(R)$ be the full and upper triangular matrix ring over $R$ separately. In a natural fashion, $\mathbb{M}_{n}(V)$, the set of all $n \times n$ matrices over $V$, is a left $\mathbb{M}_{n}(R)$ module. Similarly, $\mathbb{T}_{n}(V)$, the set of all $n \times n$ upper triangular matrices over $V$, is a left $\mathbb{T}_{n}(R)$ module. The symbol $e_{i j}, 1 \leq i, j \leq n$, will be used for a matrix having all entries zero except the $(i, j)$-entry which is equal to 1 . Note that for a module Jordan left derivation $\mu: R \rightarrow V, \mu\left(x^{2}\right)=0$ holds for all $x \in R$ if and only if $2 \mu(x)=0$ holds for all $x \in R$. The "if" part is obvious. And for all $x \in R$,

$$
2 \mu(x)=\mu(2 x)=\mu\left(x^{2}+2 x+1^{2}\right)=\mu\left((x+1)^{2}\right)=0
$$

proves the other part. For convenience, a module Jordan left derivation $\mu: R \rightarrow V$ is called strong if $\mu\left(x^{2}\right)=2 \mu(x)=0$ holds for all $x \in R$. And so, a module Jordan left derivation $\mu: R \rightarrow V$ is strong if and only if $\mu(V) \subseteq\{x \in V \mid 2 x=0\}$. Particularly, every module Jordan left derivation is strong when $V$ is 2-torsion. And the unique strong module Jordan left derivation is zero when $V$ is 2-torsion free.

Now we record some basic facts on module (Jordan) left derivations as following.

REMARK 1.1. Let $\mu: R \rightarrow V$ be a module Jordan left derivation. Then $\mu(e)=0$ for all $e=e^{2} \in R$.

Proof. By $\mu(e)=\mu\left(e^{2}\right)=2 e \mu(e)$, we have that $e \mu(e)=e(2 e \mu(e))=2 e \mu(e)$. Hence $e \mu(e)=0$, and then $\mu(e)=2 e \mu(e)=0$. 
REMARK 1.2. Let $\mu: \mathbb{M}_{n}(R) \rightarrow \mathbb{M}_{n}(V)$ (resp., $\mu: \mathbb{T}_{n}(R) \rightarrow \mathbb{T}_{n}(V)$ ) be a module Jordan left derivation. Then $\mu\left(e_{i i}\right)=0$ for all $1 \leq i \leq n$, and $\mu\left(x e_{i j}\right)=0$ for all $x \in R$ and for all $i \neq j$ (resp., $i<j$ ).

Proof. By Remark 1.1, we have $\mu\left(e_{i i}\right)=0$ for all $1 \leq i \leq n$ and $\mu\left(e_{i i}+x e_{i j}\right)=0$ for all $x \in R$ and for all $i \neq j$ (resp., $i<j)$. Hence, $\mu\left(x e_{i j}\right)=\mu\left(e_{i i}+x e_{i j}\right)-\mu\left(e_{i i}\right)=0$ for all $i \neq j$ (resp., $i<j$ ).

REMARK 1.3. Let $\mu: R \rightarrow V$ be a module left derivation. Then $x y-y x \in \operatorname{ker} \mu$ for all $x, y \in R$.

Proof. It can be proved by direct checking.

Remark 1.4. Let $\mu: R \rightarrow V$ be a strong module Jordan left derivation. Then $\mu(x y+y x)=0$ for all $x, y \in R$.

Proof. For all $x, y \in R, \mu(x y+y x)=\mu\left(x^{2}+y^{2}+x y+y x\right)=\mu\left((x+y)^{2}\right)=0$.

2. Main results. Firstly, we characterize module left derivations in full and upper triangular matrix rings over unital associative rings.

Proposition 2.1. For $n \geq 2$, a module left derivation $\mu: \mathbb{M}_{n}(R) \rightarrow \mathbb{M}_{n}(V)$ must be zero.

Proof. By Remark 1.2, $\mu\left(x e_{i j}\right)=0$ for all $i \neq j$ and for all $x \in R$. On the other hand, for all $i \neq j$ and for all $x \in R, \mu\left(x e_{i i}\right)=\mu\left(\left(x e_{i j}\right) e_{j i}\right)=\left(x e_{i j}\right) \mu\left(e_{j i}\right)+$ $e_{j i} \mu\left(x e_{i j}\right)=0$ which completes the proof. $\square$

Proposition 2.2. For $n \geq 2$, a mapping $\mu: \mathbb{T}_{n}(R) \rightarrow \mathbb{T}_{n}(V)$ is a module left derivation if and only if there exist module left derivations $\mu_{i}: R \rightarrow V(1 \leq i \leq n)$ such that for all $A=\left(a_{i j}\right) \in \mathbb{T}_{n}(R)$,

$$
\mu:\left(\begin{array}{cccc}
a_{11} & a_{12} & \cdots & a_{1 n} \\
& a_{22} & \cdots & a_{1 n} \\
& & \ddots & \vdots \\
& & & a_{n n}
\end{array}\right) \mapsto\left(\begin{array}{cccc}
\mu_{1}\left(a_{11}\right) & \mu_{2}\left(a_{11}\right) & \cdots & \mu_{n}\left(a_{11}\right) \\
& 0 & \cdots & 0 \\
& & \ddots & \vdots \\
& & & 0
\end{array}\right) .
$$

Proof. We merely deal with the "only if" part since the other part can be checked directly. By Remark 1.2, we have $\mu\left(e_{i i}\right)=0$ for all $1 \leq i \leq n$ and $\mu\left(x e_{i j}\right)=0$ for all $i<j$ and for all $x \in R$. For all $x \in R$ and for all $1 \leq i \leq n, \mu\left(x e_{i i}\right)=$ $\mu\left(e_{i i}\left(x e_{i i}\right)\right)=e_{i i} \mu\left(x e_{i i}\right)$. Particularly, for all $x \in R$ and for all $2 \leq i \leq n, 0=$ $\mu\left(x e_{1 i}\right)=\mu\left(e_{1 i}\left(x e_{i i}\right)\right)=e_{1 i} \mu\left(x e_{i i}\right)$. Hence, $\mu\left(x e_{i i}\right)=0$ for all $x \in R$ and for all $2 \leq i \leq n$ since $\mu\left(x e_{i i}\right)=e_{i i} \mu\left(x e_{i i}\right)$. For each $1 \leq i \leq n$, let $\mu_{i}: R \rightarrow V$ be the mapping such that $\mu_{i}(x)$ is the $(1, i)$-entry of $\mu\left(x e_{11}\right)$ for all $x \in R$. Obviously, 
each $\mu_{i}$ is an additive mapping. Moreover, for all $x, y \in R, \mu_{i}(x y)$ is the $(1, i)$-entry of $\mu\left(x y e_{11}\right)=x e_{11} \mu\left(y e_{11}\right)+y e_{11} \mu\left(x e_{11}\right)$ for all $1 \leq i \leq n$. And so, for each $\mu_{i}$, $\mu_{i}(x y)=x \mu_{i}(y)+y \mu_{i}(x)$ holds for all $x \in R$, which completes the proof.

By Proposition 2.2, we have the following corollaries.

COROllary 2.3. For $n \geq 2$, there exist nonzero module left derivations from $\mathbb{T}_{n}(R)$ into $\mathbb{T}_{n}(V)$ if and only if there exist nonzero module left derivations from $R$ into $V$.

Corollary 2.4. Let $V$ be an $R$-bimodule and $n \geq 2$. Then a module left derivation $\mu: \mathbb{T}_{n}(R) \rightarrow \mathbb{T}_{n}(V)$ which is also a module derivation must be zero.

If a (resp., module) left derivation is not a (resp., module) derivation, we call it nontrivial or proper. By Proposition 2.2, we can construct some nontrivial examples of (module) left derivations.

ExAmple 2.5. Let $R=\mathbb{Q}[x]$. Then for $n \geq 2$, a left derivation $\mu$ of $\mathbb{T}_{n}(R)$ must be the following form

$$
\begin{gathered}
\mu:\left(\begin{array}{cccc}
a_{11}(x) & a_{12}(x) & \cdots & a_{1 n}(x) \\
& a_{22}(x) & \cdots & a_{1 n}(x) \\
& & \ddots & \vdots \\
& & & a_{n n}(x)
\end{array}\right) \\
\mapsto\left(\begin{array}{cccc}
f_{1}(x) a_{11}^{\prime}(x) & f_{2}(x) a_{11}^{\prime}(x) & \cdots & f_{n}(x) a_{11}^{\prime}(x) \\
& 0 & \cdots & 0 \\
& & \ddots & \vdots \\
& & & 0
\end{array}\right),
\end{gathered}
$$

where $f_{1}(x), f_{2}(x), \ldots, f_{n}(x)$ are fixed polynomials in $\mathbb{Q}[x]$.

Now we characterize module Jordan left derivations in full and upper triangular matrix rings over unital associative rings.

TheOREM 2.6. For $n \geq 2$, a mapping $\mu: \mathbb{M}_{n}(R) \rightarrow \mathbb{M}_{n}(V)$ is a module Jordan left derivation if and only if there exist strong module Jordan left derivations $\mu_{i j}$ : $R \rightarrow V(1 \leq i, j \leq n)$ such that for all $A=\left(a_{i j}\right) \in \mathbb{M}_{n}(R), \mu(A)=\left(\mu_{i j}(\operatorname{tr} A)\right)$, where $\operatorname{tr} A=\sum_{i=1}^{n} a_{i i}$ is the trace of $A$. Particularly the unique module Jordan left derivation $\mu: \mathbb{M}_{n}(R) \rightarrow \mathbb{M}_{n}(V)$ is zero when $V$ is 2-torsion free.

Proof. For the "if" part, we can obtain the conclusion by Remark 1.4 and the fact that $\operatorname{tr}\left(A^{2}\right)=\sum_{i=1}^{n} a_{i i}^{2}+\sum_{i \neq j}\left(a_{i j} a_{j i}+a_{j i} a_{i j}\right)$. Now we deal with the "only if" part. By Remark 1.2, we have $\mu\left(e_{i i}\right)=0$ for all $1 \leq i \leq n$ and $\mu\left(x e_{i j}\right)=0$ for all 
$i \neq j$ and for all $x \in R$. For all $i \neq j$ and for all $x \in R$,

$$
\mu\left(x e_{i i}+x e_{j j}\right)=\mu\left(\left(e_{i j}+x e_{j i}\right)^{2}\right)=2\left(e_{i j}+x e_{j i}\right) \mu\left(e_{i j}+x e_{j i}\right)=0 .
$$

For each $1 \leq i \leq n$, using $\mu\left(e_{i i}\right)=0$, we have that

$$
\begin{aligned}
& 2 x e_{i i} \mu\left(x e_{i i}\right)=\mu\left(\left(x e_{i i}\right)^{2}\right)=\mu\left(\left((x-1) e_{i i}+I\right)^{2}\right) \\
= & 2\left((x-1) e_{i i}+I\right) \mu\left((x-1) e_{i i}+I\right)=2\left((x-1) e_{i i}+I\right) \mu\left(x e_{i i}\right) .
\end{aligned}
$$

And so, $2\left(I-e_{i i}\right) \mu\left(x e_{i i}\right)=0$ for all $x \in R$ and for all $1 \leq i \leq n$. For some $j \neq i$, we have that $0=2\left(I-e_{j j}\right) \mu\left(x e_{j j}\right)=2\left(I-e_{j j}\right) \mu\left(x e_{i i}\right)$ since $\mu\left(x e_{i i}+x e_{j j}\right)=0$. Particularly, we have that $2 e_{i i} \mu\left(x e_{i i}\right)=0$. Hence, $2 \mu\left(x e_{i i}\right)=0$ for all $x \in R$ and for all $1 \leq i \leq n$. And so, $\mu\left(x^{2} e_{i i}\right)=2 x e_{i i} \mu\left(x e_{i i}\right)=0$ for all $x \in R$ and for all $1 \leq i \leq n$. In fact, for all $i \neq j$, we have obtained $\mu\left(x e_{i i}\right)=\mu\left(x e_{j j}\right)$ for all $x \in R$. Let $\mu_{i j}: R \rightarrow V(1 \leq i, j \leq n)$ be the mapping such that $\mu_{i j}(x)$ is the $(i, j)$-entry of $\mu\left(x e_{11}\right)$ for all $x \in R$. Then $\mu_{i j}: R \rightarrow V(1 \leq i, j \leq n)$ are strong module Jordan left derivations which completes the proof.

If a (resp., module) Jordan left derivation is not a (resp., module) left derivation, we call it nontrivial or proper. By Theorem 2.6, we can construct some nontrivial examples of (module) Jordan left derivations.

ExAmple 2.7. Let $R=\mathbb{Z}_{2}[x]$, and let $f_{i j}(x) \in R(1 \leq i, j \leq n)$ be fixed polynomials. For $n \geq 2$, we obtain a nontrivial Jordan left derivation $\mu: \mathbb{M}_{n}(R) \rightarrow$ $\mathbb{M}_{n}(R)$ as $\mu(A(x))=\operatorname{tr} A(x)^{\prime}\left(f_{i j}(x)\right)$.

TheOREM 2.8. For $n \geq 2$, a mapping $\mu: \mathbb{T}_{n}(R) \rightarrow \mathbb{T}_{n}(V)$ is a module Jordan left derivation if and only if there exist module Jordan left derivations

$$
\mu_{i j}^{k}: R \rightarrow V \quad(1 \leq i \leq n, i \leq j \leq n, 1 \leq k \leq n)
$$

such that all $\mu_{i j}^{k}$ but $\mu_{1 j}^{1}(1 \leq j \leq n)$ are strong and $\mu(A)=\sum_{k=1}^{n}\left(\mu_{i j}^{k}\left(a_{k k}\right)\right)$ for all $A=\left(a_{i j}\right) \in \mathbb{T}_{n}(R)$.

Proof. It can be checked directly for the necessary part. Now we deal with the sufficient part. By Remark 1.2, we have $\mu\left(e_{i i}\right)=0$ for all $1 \leq i \leq n$ and $\mu\left(x e_{i j}\right)=0$ $(i<j)$ for all $x \in R$. Let

$$
\mu_{i j}^{k}: R \rightarrow V \quad(1 \leq i \leq n, i \leq j \leq n, 1 \leq k \leq n)
$$

be the $(i, j)$-entry of $\mu\left(x e_{k k}\right)$ for each $x \in R$. Obviously, each $\mu_{i j}^{k}$ is an additive mapping such that $\mu(A)=\sum_{k=1}^{n}\left(\mu_{i j}^{k}\left(a_{k k}\right)\right)$ for all $A=\left(a_{i j}\right) \in \mathbb{T}_{n}(R)$. Now let $x$ be an arbitrary element in $R$. For all $1 \leq i \leq n$, using $\mu\left(e_{i i}\right)=0$, we have that

$$
\begin{aligned}
& 2 x e_{i i} \mu\left(x e_{i i}\right)=\mu\left(\left(x e_{i i}\right)^{2}\right)=\mu\left(\left((x-1) e_{i i}+I\right)^{2}\right) \\
= & 2\left((x-1) e_{i i}+I\right) \mu\left((x-1) e_{i i}+I\right)=2\left((x-1) e_{i i}+I\right) \mu\left(x e_{i i}\right) .
\end{aligned}
$$


And so, $2\left(I-e_{i i}\right) \mu\left(x e_{i i}\right)=0(1 \leq i \leq n)$. This shows that $2 \mu_{i j}^{k}=0$ for all $i \neq k \in$ $\{1,2, \ldots, n\}$ and for all $i \leq j \leq n$. Particularly, for all $2 \leq i \leq n$, using $\mu\left(e_{1 i}\right)=0$, we have that

$$
\begin{aligned}
2 x e_{i i} \mu\left(x e_{i i}\right)=\mu\left(\left(x e_{i i}\right)^{2}\right) & =\mu\left(\left(x e_{i i}+e_{1 i}\right)^{2}\right) \\
=2\left(x e_{i i}+e_{1 i}\right) \mu\left(x e_{i i}+e_{1 i}\right) & =2 x e_{i i} \mu\left(x e_{i i}\right)+2 e_{1 i} \mu\left(x e_{i i}\right) .
\end{aligned}
$$

Hence, for all $2 \leq i \leq n$, we have $2 e_{1 i} \mu\left(x e_{i i}\right)=0$ which shows that $2 e_{i i} \mu\left(x e_{i i}\right)=0$. Thus, $2 \mu_{i j}^{k}=0$ for all $2 \leq k \leq n$ and for all $1 \leq i \leq j \leq n$. At the same time we have proved that $2 \mu_{i j}^{1}=0$ for all $2 \leq i \leq n$ and for all $i \leq j \leq n$. All of these shows that $\mu_{i j}^{k}\left(x^{2}\right)=0$ for all $2 \leq k \leq n$ and for all $1 \leq i \leq j \leq n$, and that $\mu_{i j}^{1}\left(x^{2}\right)=0$ for all $2 \leq i \leq n$ and for all $i \leq j \leq n$. So all $\mu_{i j}^{k}$ but $\mu_{1 j}^{1}(1 \leq j \leq n)$ are strong module Jordan left derivations. Moreover it can be checked directly that each $\mu_{1 j}^{1}(1 \leq j \leq n)$ is a module Jordan left derivation, which completes the proof.

By Theorem 2.8, we have:

Corollary 2.9. Let ${ }_{R} V$ be 2-torsion free. Then for $n \geq 2$, there exist proper module Jordan left derivations from $\mathbb{T}_{n}(R)$ into $\mathbb{T}_{n}(V)$ if and only if there exist proper module Jordan left derivations from $R$ into $V$.

By Corollary 2.9 and known results on (left) derivations in 2-torsion free prime rings $[8,11,13]$, we have:

COROLlaRY 2.10. Let $R$ be 2-torsion free prime ring. Then there is not proper module Jordan left derivations of $\mathbb{T}_{n}(R)$.

Acknowledgment. We wish to thank the referee who has clarified the concept of strong module Jordan left derivation, which is crucial to clear Jordan left derivations.

\section{REFERENCES}

[1] A. Ali and D. Kumar. Generalized derivations as homomorphisms or as anti-homomorphisms in a prime ring. Hacet. J. Math. Stat., 38:17-20, 2009.

[2] R. Alizadeh. Jordan derivations of full matrix algebras. Linear Algebra Appl., 430:574-578, 2009.

[3] M. Ashraf. On left $(\theta, \phi)$-derivations of prime rings. Arch. Math. (Brno), 41:157-166, 2005.

[4] M. Ashraf and N. Rehman. On Jordan left derivations of Lie ideals in prime rings. Southeast Asian Bull. Math., 25:379-382, 2001.

[5] M. Ashraf and S. Ali. On generalized Jordan left derivations in rings. Bull. Korean Math. Soc., 45:253-261, 2008.

[6] G.M. Benkart and J.M. Osborn. Derivations and automorphisms of non associative matrix algebras. Trans. Amer. Math. Soc., 263:411-430, 1981.

[7] D. Benkovic. Jordan derivations and antiderivations on triangular matrices. Linear Algebra Appl., 397:235-244, 2005. 
[8] M. Brešar and J. Vukman. On left derivations and related mappings. Proc. Amer. Math. Soc., 110:7-16, 1990 .

[9] S.P. Coelho and C.P. Milies. Derivations of upper triangular matrix rings. Linear Algebra Appl., 187:263-267, 1993.

[10] Q. Deng. On Jordan left derivations. Math. J. Okayama Univ., 34:145-147, 1992.

[11] I.N. Herstein. Jordan derivations of prime rings. Proc. Amer. Math. Soc., 8:1104-1110, 1957.

[12] B.E. Johnson. Continuity of derivations on commutative Banach algebras. Amer. J. Math., 91:1-10, 1969.

[13] K.W. Jun and B.D. Kim. A note on Jordan left derivations. Bull. Korean Math. Soc., 33:221228,1996

[14] Y.S. Jung. On left derivations and derivations of Banach algebras. Bull. Korean Math. Soc., 35:659-667, 1998.

[15] Y.S. Jung. Some results on Jordan left derivations in Banach algebras. Comm. Korean Math. Soc., 14:513-519, 1999.

[16] Y.S. Jung. On the generalized Hyers-Ulam stability of module left derivations. J. Math. Anal. Appl., 339:108-114, 2008.

[17] S. Jöndrup. Automorphisms and derivations of upper triangular matrices. Linear Algebra Appl., 22:205-215, 1995.

[18] S. Jöndrup. Automorphisms of upper triangular matrix rings. Arch. Math., 49:497-502, 1987.

[19] P. Mathis. Differential polynomial rings and Mortia equivalence. Comm. Algebra, 10:2001-2017, 1982.

[20] K.H. Park and Y.S. Jung. Jordan derivations and Jordan left derivations of Banach algebras. Comm. Korean Math. Soc., 17:245-252, 2002.

[21] A.M. Sinclair. Jordan homomorphisms and derivations on semisimple Banach algebras. Proc. Amer. Math. Soc., 24 :209-214, 1970.

[22] I.M. Singer and J. Wermer. Derivations on commutative normed algebras. Math. Ann., 129:260264, 1955.

[23] M.P. Thomas. The image of a derivation is contained in the radical. Ann. Math., 128:435-460, 1988.

[24] J. Vukman. On left Jordan derivations of rings and Banach algebras. Aequationes Math., 75:260-266, 2008.

[25] X.W. Xu and H.Y. Zhang. A note on generalized left $(\theta, \phi)$-derivations in prime rings. Hacet. J. Math. Stat., to appear.

[26] S.M.A. Zaidi, M. Ashraf, and S. Ali. On Jordan ideals and left $(\theta, \theta)$-derivations in prime rings. Int. J. Math. Math. Sci., 37:1957-1964, 2004. 